\title{
Projective metabelian nonfree groups
}

\section{V.A. Artamonov}

This paper is concerned with projective metabelian $\underline{\underline{A A}}$-groups, where $A$ is the variety of all abelian groups, $\frac{A}{A n}$ - of all abelian groups of exponent $n$. Let $P$ be a projective $\stackrel{A A}{=}{ }^{-}$ group. Since $\stackrel{A A}{A} \supset$ the group $P / P^{\prime}$ is a free abelian group. Define rank $P=\operatorname{rank} P / P^{\prime}$. It is shown that for all numbers $r, n>1$, except $r=n=2$, there exists a projective nonfree $\stackrel{A A}{n}$-group of rank $r$ with $r+1$ generators.

\section{Introduction}

This paper is concerned with projective metabelian ${ }_{n}$-groups, where $A$ is the variety of all abelian groups, $\underline{A}_{n}$ - of all abelian groups of

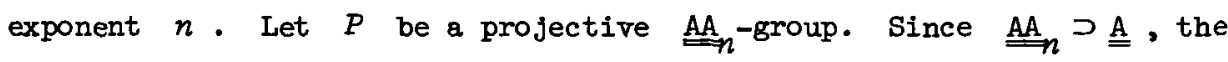
group $P / P^{\prime}$ is a free abelian group. Define rank $P=\operatorname{rank} P / P^{\prime}$. We show that if $r, n \geq 2$, except $r=n=2$, then there exists a projective nonfree AA-group of rank $r$ with $r+1$ generators. On the other hand, Mclsaac [4] has proved that projective $A A$-groups of rank 2 are free. It is not difficult to prove that projective $\underline{\underline{A A}}$-groups of rank 1 are free.

\section{Preliminaries}

Let $c_{r, n}$ be a direct product of $r$ cyclic groups $\left\{x_{i}\right\}$ of the same

Received 28 April 1975. The author thanks Dr P.M. Neumann, Mr A.J. Mclsaac, and Professor A.L. Smel'kin for reading this paper and for useful discussions. 
order $n$. Consider the group ring $Z C_{r, n}$. Let $m$ be the augmentation ideal of $Z C_{r, n}$. For $y \in C_{r, n}$ define $n(y)=1+y+\ldots+y^{n-1}$.

PROPOSITION 1. If $N_{r, n}=\sum_{y \in C_{r, n}} y \in Z C_{r, n}$, then $N_{r, n}=\prod_{i=1}^{r} n\left(x_{i}\right)$

Proof. For all $x_{j}, 1 \leq j \leq r$, we have $x_{j} \prod_{i=1}^{r} n\left(x_{i}\right)=\prod_{i=1}^{r} n\left(x_{i}\right)$. So $\prod_{i=1}^{r} n\left(x_{i}\right)=a N_{r, n}, a \in Z$, since only $Z N_{r, n}$ satisfies this property. But $a N_{r, n} \equiv a n^{r}(\bmod m) \equiv \prod_{i=1}^{r} n(1)=n^{r} \quad$ and, hence, $a=1$. Consider now the augmentation epimorphism

$$
\varepsilon: Z C_{r, n}+Z, \varepsilon\left(x_{i}\right)=1, \operatorname{ker} \varepsilon=m \text {. }
$$

It induces an epimorphism

$$
\varepsilon^{\prime}: Z C_{r, n} /\left(N_{r, n}\right)+\mathrm{Z} /\left(n^{r^{2}}\right),
$$

since $N \equiv n^{r}(\bmod m)$. In its turn $\varepsilon^{\prime}$ induces a homomorphism of groups of units

$$
\varepsilon^{*}=\varepsilon_{r, n}:\left(Z C_{r, n^{\prime}} /\left(N_{r, n}\right)\right) * \rightarrow\left(\mathrm{Z} /\left(n^{r^{2}}\right)\right) *
$$

THEOREM 1. Let $r, n \geq 2$ except $r=n=2$. Then $\varepsilon_{r, n}$, from (1), is not an epimorphism.

Proof. Suppose that for $r-1$ the theorem is proved, and $K+\left(n^{r-1}\right) \in\left(Z / n^{r-1}\right) * \backslash \operatorname{Im} \varepsilon_{r-1, n}$. Then $K+\left(n^{r}\right) \in\left(Z / n^{r}\right) *$. Suppose $K+\left(n^{r}\right) \in \operatorname{Im} \varepsilon_{r, n}$. Then $K \equiv f\left(x_{1}, \ldots, x_{r}\right)\left(\bmod \left(m, n^{p}\right)\right)$, where $f\left(x_{1}, \ldots, x_{r}\right) \in Z C_{r, n}$ and, for some $g\left(x_{1}, \ldots, x_{p}\right) \in Z C_{r, n}$, 
(2) $\quad f\left(x_{1}, \ldots, x_{p}\right) g\left(x_{1}, \ldots, x_{r}\right)=1+a N_{r, n}=1+a \prod_{i=1}^{r} n\left(x_{i}\right)$. Put $x_{p}=1$ in (2). We have

$f\left(x_{1}, \ldots, x_{r-1}, 1\right) g\left(x_{1}, \ldots, x_{r-1}, 1\right)=1+n a \prod_{i=1}^{r-1} n\left(x_{i}\right)=1+$ naN $_{r-1, n}$. So $f\left(x_{1}, \ldots, x_{r-1}, 1\right) \in\left(Z C_{r-1, n} / N_{r-1, n}\right)^{*}$,

$$
f\left(x_{1}, \ldots, x_{r-1}, 1\right) \equiv f\left(x_{1}, \ldots, x_{r}\right) \equiv k\left(\bmod \left(m, n^{r}\right)\right)
$$

and $K+\left(n^{r-1}\right) \in \operatorname{Im} \varepsilon_{r-1, n}$. This contradiction shows that $K+\left(n^{r}\right) \notin \operatorname{In} \varepsilon_{r-1, n}$

This remark shows that we need only to prove that $\varepsilon_{3,2}$ and $\varepsilon_{2, n}$, $n>2$, are not epimorphisms.

LEMMA 1. $\varepsilon_{3,2}$ is not an epimorphism.

Proof. Let $A=A(x, y, z), B=B(x, y, z) \in Z_{3,2}$ and $A B=1+a N_{3,2}, a \in Z$. By Proposition $1, A(-1, y, z)$ is a unit in $Z c_{2,2} \subset Z C_{3,2}$. But all units in $Z c_{2,2}$ are trivial (see [2], Theorem 6). Since $\varepsilon_{3,2}$ maps trivial units into \pm 1 we can assume that $A(-1, y, z)=B(-1, y, z)=1$. Then $x^{2}=y^{2}=z^{2}=1$ implies $A=1+(1+x)\left(a_{0}+a_{1} y+a_{2} z\right)$. So,

$$
A(x,-1, z)=\left(1+a_{0}-a_{1}\right)+x\left(a_{0}-a_{1}\right)+a_{2} z+a_{2} x z .
$$

Again, by Higman's Theorem, two of the three numbers $1+a_{0}-a_{1}$, $a_{0}-a_{1}, a_{2}$ equal zero. But $1+a_{0}-a_{1} \neq a_{0}-a_{1}$. Hence $a_{2}=0$. Similarly, the consideration of $A(x, y,-1)$ implies $a_{1}=0$. Thus $A=1+a_{0}(1+x)$. The same argument shows $B=1+b_{0}(1+x)$. Hence $A B=1+\left(a_{0}+b_{0}+2 a_{0} b_{0}\right)(1+x)=1+a(1+x)(1+y)(1+z)$ and this implies $a_{0}+b_{0}+2 a_{0} b_{0}=0$. If $a_{0}=0$ then $b_{0}=0$ and $A=1, \varepsilon_{3,2}(A)=1$. 
Otherwise $a_{0}=b_{0}=1$ and $A=-x, \varepsilon_{3,2}(A)=-1$. So, in all cases, Im $\varepsilon_{3,2}=\{1,-1\} \neq\{1,3,5,-1\}=(2 / 8)^{*}$. This proves Lemma 1 .

Suppose now $r=2, n>2, n=a b$. Then there is a natural epimorphism $f: Z c_{2, n} \rightarrow Z C_{2, b}$. It is clear that $f\left(n\left(x_{i}\right)\right)=a \cdot b\left(x_{i}\right)$; hence $f\left(N_{2, n}\right)=a^{2} N_{2, b}$. The epimorphism $f$ induces a homomorphism rings $f^{\prime}: Z C_{2, n} / N_{2, n}+Z C_{2, b} / N_{2, b}$ and a homomorphism of groups $f^{*}:\left(\mathrm{ZC}_{2, n^{\prime}} / N_{2, n}\right)^{*} \rightarrow\left(\mathrm{Z} C_{2, b} / N_{2, b}\right)^{*}$. Let $j$ be the natural homomorphism $j: Z / n^{2}+Z / b^{2}, j\left(K+\left(n^{2}\right)\right)=K+\left(b^{2}\right)$.

LEMMA 2. The following diagram

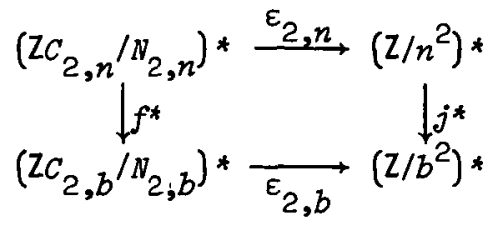

is commutative.

The proof is trivial, since all homomorphisms are induced by ring homomorphisms and for ring generators $x_{i}$ we have $j^{*} \varepsilon_{2, n^{x} i}=1+\left(b^{2}\right)=\varepsilon_{2, b^{*} x_{i}}$.

COROLLARY. If $\varepsilon_{2,4}$ is not epi, then $\varepsilon_{2,2^{k}}, K \geq 2$, is not epi either.

The proof follows from Lemma 2 , since $j^{*}$ is epi.

LEMMA 3. $\varepsilon_{2,4}$ is not epi.

Proof. We shall show that $3+(16)$ Im $\varepsilon_{2,4}$. Let $A=A(x, y)$, $B=B(x, y) \in Z C_{2,4}$, and $A B=1+c N_{2,4}, c \in Z ;$ put $x=-1$. Since $(1+x) \mid N_{2,4}$ we have in $Z C_{1,4}$,

$$
A(-1, y) B(-1, y)=1 \text {. }
$$

By Theorem 6 from [2], $Z C_{1,4}$ contains only trivial units, so, without loss of generality, we suppose $A(-1, y)=1$; 
that is, $A(x, y)=1+(1+x) D(x, y)$ where $D(x, y)=E(x)+(1+y) J(x, y)$. By Theorem 6 from [2], $A(x,-1)=1+(1+x) E(x)$ is a trivial unit, $A(x,-1)= \pm x^{t}$. Thus $A(x, y)= \pm x^{t}(1+(1+x)(1+y) J(x, y))$. Let $J(x, y)=J_{1}(x)+y J_{2}(x)+\left(1+y^{2}\right) J_{3}(x, y)$. Then in $Z[i] C_{1,4}$,

$$
\pm x^{4-t} A(x, i)=1+(1+x)\left(J_{1}(x)-J_{2}(x)\right)+i(1+x)\left(J_{1}(x)+J_{2}(x)\right)
$$

is a unit. But $Q[i] C_{1,4}$ is isomorphic to the direct sum of four copies of $\mathrm{Q}[i]$ in which the generator of $C_{1,4}$ acts as a multiplication by $\pm 1, \pm i$. So, since all units of $Q[i]$ have finite order, the same is true by Theorem 5 from [2] for $Z[i] C_{1,4}$, and by Theorem 3 (see [2]) a]l units of $Z[i] C_{1,4}$ are of the form $\pm x^{k}, \pm i x^{k}$. Hence, in (2'), $J_{1}=J_{2}=0$, and $A(x, y)= \pm x^{t}\left[1+(1+x)(1+y)\left(1+y^{2}\right) J_{3}(x, y)\right]$. Similarly, $J_{3}(x, y)=\left(1+x^{2}\right) J_{4}$ and thus $A(x, y) \equiv \pm x^{t}\left(\bmod N_{2,4}\right), \varepsilon_{2,4}(A)= \pm 1$.

Suppose now that $n=p^{k} d, k \geq 1, p$ an odd prime, and we have already proved that for all $z \in \operatorname{Im} \varepsilon_{2, p}$,

$$
z^{2(p-1)^{2}}=1
$$

Then $1+p d+\left(n^{2}\right) \mid \operatorname{Im} \varepsilon_{2, n}$. Indeed, if $1+p d+\left(n^{2}\right) \in \operatorname{Im} \varepsilon_{2, n}$, then by Lemma $2,1+p d+\left(p^{2}\right) \in \operatorname{Im} \varepsilon_{2, p}$, so $\bmod p^{2}$,

$$
(1+p d)^{2(p-1)^{2}}-1 \equiv(1+2 p d)-1 \equiv 2 p d \equiv 0 ;
$$

this contradicts $(d, p)=(2, p)=1$. Thus, we need only prove (3).

Let $H$ be the group of all automorphisms of $\mathrm{Z}_{2, p}=\mathrm{Z}[x, y] /\left(x^{p}-1, y^{p}-1\right)$ leaving invariant the cyclic subgroups $\{x\},\{y\} \subset C_{2, p}$. It is clear that for $\alpha \in H, g \in Z C_{2, p}$,

$$
\varepsilon(\alpha(g))=\varepsilon(g)
$$

and $|B|=(p-1)^{2}$. Note that the element $N=N_{2, p}$ is $H$-invariant; 
that is, for all $\alpha \in H$,

$$
\alpha(N)=N \text {. }
$$

LEMMA 4. Let $A \in Z C_{2, p}$ be $H$-invariant, $B \in Z C_{2, p}$. If $A B \equiv 1(\bmod N)$ then $B$ is $H$-invariant.

$$
\begin{aligned}
& \text { Proof. If } A B=1+\alpha N, \alpha \in Z \text {, then } \\
& 0=\alpha(1+a N)-(1+a N)=A[\alpha(B)-B], \alpha \in H .
\end{aligned}
$$

Since $A$ is invertible $\bmod N$,

$$
\alpha(B)=B+b N, b \in \mathrm{Z},
$$

and, by induction, $\alpha^{k}(B)=B+k b N$. But for some $k \geq 1$ we have $\alpha^{k}=1$, so $k b N=0$ and $b=0$.

LEMMA 5. Let $c \in Z_{2, p}$ and $c$ be H-invariant. Then $C=c_{0}+c_{1} p(x)+c_{2} p(y)+c_{3} p(x) p(y)$ where $c_{i} \in Z$.

Proof. Let $C=\sum_{i, j=0}^{p-1} a_{i j} x^{i} y^{j}$. For each $i, 1 \leq i \leq p-1$, we have an automorphism $\beta \in H$ such that $\beta(x)=x^{i}, \beta(y)=y$. Since $C$ is invariant, $a_{i j}=a_{1 j}, 1 \leq i \leq p-1$. The same is true for $j$. This proves Lemma 5.

LEMMA 6. If $z \in \operatorname{Im} \varepsilon_{2, p}$, then $z^{2(p-1)^{2}}=1$.

Proof. Since for all $A \in Z C_{2, p}, \alpha \in H, \quad \varepsilon(\alpha(A))=\varepsilon(A)$ and $|H|=(p-1)^{2}$, we need only prove that for $H$-invariant $A$, invertible $\bmod N$,

$$
\varepsilon_{2, p}(A+(N))= \pm 1 \text {. }
$$

By Lemma 4, for $H$-invariant $B \in Z C_{2, p}$,

$$
A B=1+a N, \quad a \in Z \text {. }
$$

By Lemma 5 we can assume $A=a_{0}+a_{1} p(x)+a_{2} p(y)$, $B=b_{0}+b_{1} p(x)+b_{2} p(y)$. Note that $p(x)^{2}=p \cdot p(x) ;$ so in $Z c_{2, p}$, 


$$
\begin{aligned}
A B=a_{0} b_{0}+\left(a_{0} b_{1}+a_{1} b_{0}+p a_{1} b_{1}\right) p(x) & +p(y)\left(a_{0} b_{2}+a_{2} b_{0}+p a_{2} b_{2}\right)+ \\
& +p(x) p(y)\left(a_{1} b_{2}+a_{2} b_{1}\right)=1+a p(x) p(y),
\end{aligned}
$$

which implies $a_{0} b_{1}+a_{1} b_{0}+p a_{1} b_{1}=a_{0} b_{2}+a_{2} b_{0}+p a_{2} b_{2}=0, a_{0} b_{0}=1$. Hence $a_{0}=b_{0}= \pm 1, b_{1}= \pm a_{1}\left( \pm 1+p b_{1}\right)$, and

$$
\left|b_{1}\right| \geq\left|p b_{1} \pm 1\right| \geq p\left|b_{1}\right|-1 \geq 2\left|b_{1}\right| \text {, }
$$

since $p \geq 3$. Thus $b_{1}=a_{1}=0$. Similarly $a_{2}=b_{2}=0$. Hence, finally, $A= \pm 1$. This completes the proof of Theorem 1 .

THEOREM 2. Let the numbers $r, n$ be as in Theorem 1. Then there exists a projective nonfree $Z C_{r, n}$-module $T$ of rank $r$ with epimorphism $I: T \rightarrow m$ (the augmentation ideal of $\mathrm{ZC}_{r, n}$ ) such that

(i) for some projective ideal $J \triangleleft Z C_{r, n}$ the module $M=J \oplus T$ is free of rank $r+1$,

(ii) if $Z$ is trivially extended to $M$, so that $Z(J)=0$. and if $\rho$ is a projection of $M$ onto $T$, then for some basis $w_{0}, \ldots, w_{p}$ of $M$,

$$
\begin{array}{ll}
\rho\left(w_{0}\right) \in m T, & \tau\left(w_{0}\right)=0, \\
\rho\left(w_{i}\right) \equiv w_{i}(\bmod m I), & \tau\left(w_{i}\right)=x_{i}-1, \quad I \leq i \leq r .
\end{array}
$$

Proof. Let $k \in Z$ and $k+\left(n^{r}\right) \in\left(Z / n^{r}\right) * \backslash \operatorname{Im} \varepsilon_{r, n}$. Then $(k, n)=1$ and for some $k^{\prime}, m \in Z$,

$$
k k^{\prime}=1+m n^{r} \text {. }
$$

By results from [6] the ideals $I=(k, N), J=\left(k^{\prime}, N\right)$, where $N=N_{r, n}$, are projective nonfree $Z C_{r, n}$-modules of rank 1 . There exists an isomorphism

$$
\begin{gathered}
f:\left(\mathrm{Z} c_{r, n}\right)^{2} \rightarrow J \oplus I, \\
f(1,0)=\left(k u^{\prime}-m v^{\prime}, u\right), f(0, I)=\left(m n^{r} u^{\prime}-m k^{\prime} v^{\prime}, k^{\prime} u-m v\right),
\end{gathered}
$$


where $u^{\prime}=k^{\prime}, v^{\prime}=N \in J, u=k, v=N \in I$. Take in $\left(Z c_{r, n}\right)^{2}$ a new basis $e_{0}=k(1,0)-(0,1), e_{1}=(1,0)$. Then by (4),

$$
f_{0}=f\left(e_{0}\right)=\left(u^{\prime}, m v\right), f_{1}=f\left(e_{1}\right)=\left(k u^{\prime}-m v^{\prime}, u\right) \text {. }
$$

In [6] Swan noticed that $I=(k, N)$ is given by generators $u, v$ and relations

$$
N u=k v, x v=v \text {, for all } x \in C_{r, n} .
$$

Thus, if $Z: I \rightarrow m$ is any homomorphism, then $k Z(v)=N Z(u)=0$ by Proposition 1 , since $Z(u)=\sum a_{i}\left(x_{i}-1\right)$. So, by (4"), all homomorphisms $\tau: I \rightarrow m$ are uniquely determinded by $Z(u)$, and since $Z C_{r, n} u \simeq Z C_{r, n}$ there is no restriction on $Z(u)$.

Now take $T=I \oplus Z C_{r, n} f_{2} \oplus \ldots \oplus Z_{r, n} f_{r}$.

LEMMA 7. $T$ is a projective nonfree $\mathrm{Z} C_{r, n}$-module of rank $r$.

Proof. If $T \simeq\left(Z c_{r, n}\right)^{r}$, then since $K r u l l-\operatorname{dim} Z C_{r, n}=1$ (see [1], p. 600) by the 'cancellation' theorem ([1], p. 184$), I \oplus Z C_{r, n}=\left(Z C_{r, n}\right)^{2}$. But $G L\left(2, Z C_{r, n}\right)$ acts transitively on the set of all unimodular vectors in $Z C_{r, n}$. So $I \simeq Z C_{r, n}$ (see [3], p. 286). This contradicts $I \neq Z C_{r, n}$.

LEMMA 8. Let

$$
\begin{aligned}
& A_{0}=-m n^{r-1} n(x)+k k^{\prime} x+(y-1), \\
& A_{1}=k^{\prime} m m^{r-1} n(x)-k^{\prime} m n^{r} x-k^{\prime}(y-1), \\
& A_{3}=x-1 .
\end{aligned}
$$

Then

$$
\begin{aligned}
& \text { (i) } A_{0} \equiv 1(\bmod m), A_{2}, A_{3} \in m, \\
& \text { (ii) }\left(m m^{r} A_{0}+k A_{1}\right)(x-1)+A_{2}(y-1)=0,
\end{aligned}
$$


(iii) the ideal $L=\left(A_{0}, A_{1}, A_{2}\right)=Z C_{r, n}$.

Proof. Since $A_{i} \equiv A_{i}(1,1)(\bmod m)$, (4) implies $A_{0}(1,1)=-n n^{r}+k k^{\prime}=1, A_{1}(1,1)=k^{\prime} m n^{r}-k^{\prime} m n^{r}=0, A_{2}(1,1)=0$. Thus (i) is proved.

$$
\begin{aligned}
& \text { Now } n(x)(x-1)=0 \text {, so } \\
& {\left[m n^{r} A_{0}+k A_{1}\right](x-1)+A_{2}(y-1)=} \\
& \quad=\left[m n^{r}\left(k k^{\prime} x+(y-1)\right)+k\left(-k^{\prime} m n^{r} x-k^{\prime}(y-1)\right)\right](x-1)+(x-1)(y-1)=0 .
\end{aligned}
$$

Since $L \rightarrow x-1$ by $(i), A_{0} \equiv I+(y-1) \equiv Y(\bmod L)$ and $1 \in L$.

LEMMA 9. There exists $A \in \mathrm{GL}\left(3, Z C_{2, n}, m\right)$ such that the first row of $A$ is $A_{0}, A_{1}, A_{2}$.

Proof. Since Krull-dim $Z c_{r, n}=1$ by results of Chapter 5 of [1], there exists $C \in \mathrm{GL}\left(3, Z C_{r, n}\right)$ with the first row $c_{00}=A_{0}, c_{01}=A_{1}$, $c_{02}=A_{2}$. But $A_{0} \equiv 1(\bmod m)$, so, applying elementary transformations to $C$, we can suppose $c_{10}, c_{20} \in m$. Now mod $m$,

$$
c \equiv C_{0}=\left(\begin{array}{lll}
i & 0 & 0 \\
0 & a & b \\
0 & c & d
\end{array}\right),\left(\begin{array}{ll}
a & b \\
c & d
\end{array}\right) \in \operatorname{GL}(2, Z),
$$

and we can take $A$ to be $A=C_{0}^{-1} C \in \mathrm{GL}\left(3, \mathrm{Z} C_{r, n}, m\right)$.

Note that

$$
\left|\begin{array}{cc}
k^{\prime} & m n^{r} \\
1 & k
\end{array}\right|=I \text {, }
$$

so 


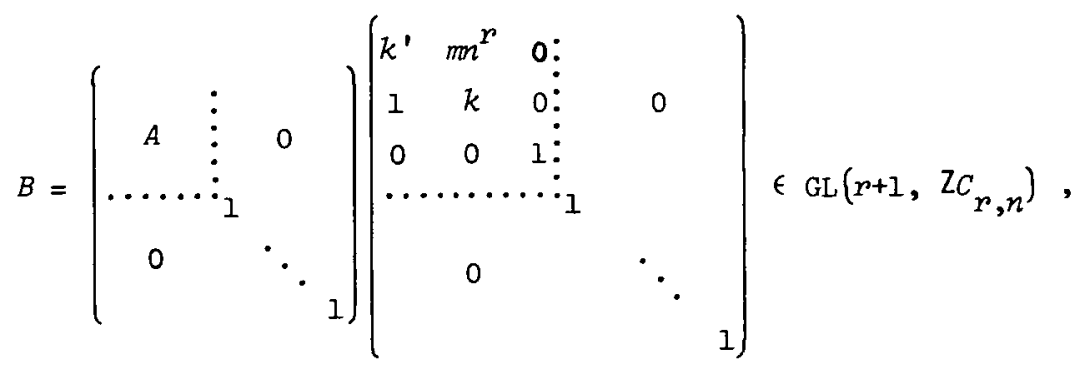

where $A$ is from Lemma 9. Put $w_{i}=B^{-1} f_{i}, i=0, \ldots, r$, and define $Z\left(W_{0}\right)=0, \quad Z\left(W_{i}\right)=x_{i}-1, i=1, \ldots, r$. We need to prove that $Z(J)=0$ and $W_{0}, \ldots, W_{p}$ satisfy $(i i)$ from Theorem 2 .

LEMMA 10. $Z(J)=0$.

Proof. Since $Z(J)=Z C_{r, n} Z\left(u^{\prime}\right)$, we need to prove, by (4'), that $2\left(f_{0}\right)=0$. But $f_{0}=b_{00} W_{0}+b_{01} W_{1}+b_{02} W_{2}$, $Z\left(f_{0}\right)=\left(A_{0} m n^{r}+A_{1} k\right)(x-1)+A_{2}(y-1)=0$ by (ii) from Lemma 8 .

LEMMA 11. If $\rho$ is a projection $\rho: M \rightarrow T$, then $\rho\left(W_{0}\right) \in m I$, $\rho\left(w_{i}\right) \equiv w_{i}(\bmod m M), i=1, \ldots, r$.

Proof. Since $A \in \mathrm{GL}\left(3, \mathrm{Z}_{r, n}, m\right)$ and $w_{i} \equiv f_{i}=\rho\left(f_{i}\right)(\bmod m M)$, $2 \leq i \leq r$, without loss of generality we suppose $A=1, r=2$. Then

$$
B^{-1}=\left(\begin{array}{ccccc}
k & -m n^{r} \vdots & & \\
& \vdots & 0 & \\
-1 & k^{\prime} & \vdots & \\
\cdots & \ldots & \cdots & & \\
0 & & \ddots & \\
& & & & 1
\end{array}\right) \text {, }
$$

$$
\begin{aligned}
& w_{0}=k f_{0}-m m^{r} f_{1}, w_{1}=-f_{0}+k^{\prime} f_{1} . \text { By }(4),\left(4^{\prime}\right) \text {, } \\
& \rho\left(w_{0}\right)=k m v-m m^{r} u=m\left(N-n^{r}\right) u \in m u, \\
& w_{1}-\rho\left(w_{1}\right)=\left(-u^{\prime},-m v\right)+k^{\prime}\left(k u^{\prime}-m v^{\prime}, u\right)+(0, m v)-k^{\prime}(0, u)= \\
& =m\left(\left(N-n^{r}\right) u^{\prime}, 0\right) \in m J \text {. }
\end{aligned}
$$




$$
\text { Projective metabelian nonfree groups }
$$

This completes the proof of Theorem 2 .

\section{Projective $\underline{\underline{A A}}_{n}$-groups}

Let $P$ be a projective $\underline{\underline{\mathrm{AA}}}$-group. As we have already noticed in the introduction, rank $P=\operatorname{rank} P / P^{\prime}$.

THEOREM 3. Let $r, n \geq 2$ except $r=n=2$. Then there exist projective nonfree $\stackrel{\mathrm{AA}}{=}$-groups of rank $r$ with $r+1$ generators.

Proof. Let $Z C_{r, n}=Z\left[x_{1}, \ldots, x_{r}\right] /\left(x_{i}^{n}-1, i=1, \ldots, r\right)$ with augmentation ideal $m, Z C_{p+1, n}=\mathrm{Z}\left[x_{0}, \ldots, x_{p}\right] /\left(x_{i}^{n}-1, i=0, \ldots, r\right)$ with augmentation ideal $m_{0}$, and $T, l, J, w_{i}$ from Theorem 2 . Let

$$
S=Z C_{r+1, n^{2}} \oplus \ldots \oplus Z C_{r+1, n^{W}}
$$

Define $z_{0}: S \rightarrow m_{0}$ by $z_{0}\left(w_{i}\right)=x_{i}-1$.

In [5] it is shown that the group $F$ of matrices

$$
\left(\begin{array}{ll}
a & 0 \\
b & 1
\end{array}\right), a \in C_{p+1, n}, b \in S, a-1=Z(b),
$$

is a free AA-group with $r+1$ free generators

$$
z_{i}=\left(\begin{array}{ll}
x_{i} & 0 \\
w_{i} & 1
\end{array}\right)
$$

Note that for $t_{i}=\left(\begin{array}{ll}a_{i} & 0 \\ b_{i} & 1\end{array}\right) \in F$,

$$
\begin{aligned}
& t_{1} t_{2}=\left(\begin{array}{cc}
a_{1} a_{2} & 0 \\
a_{2} b_{1}+b_{2} & 1
\end{array}\right), t_{1}^{-1}=\left(\begin{array}{cc}
a_{1}^{-1} & 0 \\
-a_{1}^{-1} b_{1} & 1
\end{array}\right), \\
& t_{1}^{t_{2}}=\left(\begin{array}{cc}
a_{1} & 0 \\
a_{2} b_{1}-\left(a_{1}-1\right) b_{2} & 1
\end{array}\right), t_{1}^{n}=\left(\begin{array}{cc}
1 & 0 \\
n\left(a_{1}\right) b_{1} & 1
\end{array}\right) .
\end{aligned}
$$


Let $\varphi$ be an endomorphism of $F$,

$$
\varphi\left(z_{i}\right)=\left(\begin{array}{cc}
a_{i} & 0 \\
\Phi W_{i} & 1
\end{array}\right), \Phi=\Phi\left(x_{0}, \ldots, x_{p}\right) \in M a^{t}\left(p+1, Z C_{p+1, n}\right) .
$$

LEMMA 12. If

$$
z=\left(\begin{array}{cc}
a\left(x_{0}, \ldots, x_{p}\right) & 0 \\
\sum b_{i}\left(x_{0}, \ldots, x_{p}\right) w_{i} & 1
\end{array}\right) \text {, }
$$

then

$$
\varphi(z)=\left(\begin{array}{cc}
a\left(a_{0}, \ldots, a_{p}\right) & 0 \\
\sum b_{i}\left(a_{0}, \ldots, a_{p}\right) \Phi W_{i} & 1
\end{array}\right) .
$$

The proof is trivial, since (6) is valid for $z_{0}, \ldots, z_{p}$, and if (6) is valid for $t_{1}, t_{2}$, then, by (5), it is valid for $t_{1} t_{2}, t_{1}^{-1}$.

LEMMA 13. Let $\varphi$ be an endomorphism of $F$ such that

$$
\varphi\left(z_{0}\right)=\left(\begin{array}{cc}
1 & 0 \\
\Phi W_{0} & 1
\end{array}\right), \quad \varphi\left(z_{i}\right)=\left(\begin{array}{cc}
x_{i} & 0 \\
\Phi W_{i} & 1
\end{array}\right), \quad i=1, \ldots, r .
$$

Then

$$
U=\left\{\left(\begin{array}{cc}
1 & 0 \\
{\left[\begin{array}{ll}
b_{i} W_{i} & 1
\end{array}\right) \in F} & b_{i} \in\left(x_{0}-1\right)
\end{array}\right\} \triangleleft F\right.
$$

and $U \subseteq \operatorname{ker} \varphi$.

Proof. By (5), $U \triangleleft F$ and by (6), $U \subseteq \operatorname{ker} \varphi$, since $a_{0}=1$, $b_{i}\left(1, x_{1}, \ldots, x_{p}\right)=1$.

Now we can prove the theorem. We have $M \subset S$. Let $\rho$ be the projection $\rho: M \rightarrow T, \rho=\left(\rho_{i j}\right)$. By (ii) from Theorem 2, $\rho_{i 0}=\sum_{j=1}^{r} \alpha_{i j}\left(x_{j}-1\right)$. Define 
(7)

$$
\Phi_{i j}= \begin{cases}\rho_{i 0} & , j=0, \\ \rho_{i j}-\alpha_{i j}\left(x_{0}-1\right), & j \geq 1 .\end{cases}
$$

Then

$$
\Phi_{i j} \equiv \rho_{i j}\left(\bmod \left(x_{0}-1\right)\right), \Phi=\left(\Phi_{i j}\right)
$$

Put

$\left(7^{\prime \prime}\right)$

$$
\varphi\left(z_{i}\right)= \begin{cases}\left(\begin{array}{cc}
1 & 0 \\
\Phi W_{0} & 1
\end{array}\right), \quad i=0, \\
\left(\begin{array}{ll}
x_{i} & 0 \\
\Phi W_{i} & 1
\end{array}\right), \quad i \geq 1 .\end{cases}
$$

LEMMA 14. $\varphi\left(z_{i}\right) \in F$.

Proof.

$$
\begin{array}{r}
\tau_{0}\left(\Phi W_{0}\right)=\sum_{j=0}^{r} \Phi_{0 j}\left(x_{j}-1\right)=\sum_{j=1}^{r} \alpha_{0 j}\left(x_{j}-1\right)\left(x_{0}-1\right)+\sum_{j=1}^{r}\left(\rho_{0 j}-\alpha_{0 j}\left(x_{0}-1\right)\right)\left(x_{j}-1\right)= \\
=\sum_{j=1}^{r} \rho_{0 j}\left(x_{j}-1\right)=2\left(\rho\left(W_{0}\right)\right)=2\left(W_{0}\right)=0
\end{array}
$$

since $Z(\operatorname{ker} \rho)=Z(J)=0$. Similarly for $i \geq 1$,

$$
\begin{array}{r}
\tau_{0}\left(\Phi W_{i}\right)=\sum_{j=0}^{r} \Phi_{i j}\left(x_{j}-1\right)=\sum_{j=1}^{r} \alpha_{i j}\left(x_{j}-1\right)\left(x_{0}-1\right)+\sum_{j=1}^{r}\left[\rho_{i j}-\alpha_{i j}\left(x_{0}-1\right)\right]\left(x_{j}-1\right)= \\
=\sum_{j=1}^{r} \rho_{i j}\left(x_{j}-1\right)=2\left(\rho\left(w_{i}\right)\right)=2\left(w_{i}\right)=x_{i}-1 .
\end{array}
$$

Thus $\varphi \in$ end $F$.

LEMMA 15. Let $\pi=\varphi^{2} ;$ then $\pi^{2}=\pi$.

Proof. We have 


$$
\varphi\left(z_{0}\right)=\left(\begin{array}{cc}
1 & 0 \\
\rho\left(W_{0}\right)+\left(x_{0}-1\right) y_{0} & 1
\end{array}\right), \varphi\left(z_{i}\right)=\left(\begin{array}{cc}
x_{i} & 0 \\
\rho\left(W_{i}\right)+\left(x_{0}-1\right) y_{i} & 1
\end{array}\right) \text {, }
$$

where $y_{i} \in M \subset S$. Then by (6), ( $\left.7^{\prime}\right),(5)$, and $\rho^{2}=\rho$ we have $\varphi^{2}\left(z_{i}\right) \equiv \varphi\left(z_{i}\right)(\bmod U)$ where $U$ is a normal subgroup from Lemma 13. Hence $\pi\left(z_{i}\right)=\varphi^{2}\left(z_{i}\right)=\varphi\left(z_{i}\right) u_{i}, u_{i} \in U \subseteq \operatorname{ker} \varphi$ and

$$
\pi^{2}\left(z_{i}\right)=\varphi^{4}\left(z_{i}\right)=\varphi^{3}\left(z_{i}\right)=\varphi^{2}\left(z_{i} u_{i}\right)=\varphi^{2}\left(z_{i}\right)=\pi\left(z_{i}\right) \text {. }
$$

Put $P=\operatorname{Im} \pi$. Then $P$ is a projective $\stackrel{\mathrm{AA}}{=}$-group with $r+1$ generators.

LEMMA 16. rank $P=r$.

Proof. Let

$$
G=\left\{\left(\begin{array}{ll}
l & 0 \\
g & I
\end{array}\right) \in F\right\} .
$$

Then, by (5) and (6), $G$ is a verbal subgroup of $F$ corresponding to the variety $\stackrel{A}{n}_{n}$. By $\left(7^{\prime \prime}\right), P / P \cap G$ is a free $Z /(n)$-module of rank $r$. But $r=\operatorname{rank} P / P \cap G=\operatorname{rank} P / P^{\prime}=\operatorname{rank} P$.

Suppose that $P$ is a free $A A_{n}$-group. Since every automorphism of $P / P^{\prime}$ can be lifted to an automorphism of $P$ (see [5]) we can choose in $P$ free generators $t_{1}, \ldots, t_{p}$ such that in $F$,

$$
t_{i}=\left(\begin{array}{ll}
x_{i} & 0 \\
g_{i} & 1
\end{array}\right), g_{i} \in S .
$$

Let $g_{i}=g_{i}^{\prime}+\left(x_{0}-1\right) g_{i}^{\prime \prime}$, where $g_{i}^{\prime} \in M, g_{i}^{\prime \prime} \in S$. Since $\pi\left(t_{i}\right)=t_{i}$, (6), ( $\left.7^{\prime}\right),\left(7^{\prime \prime}\right)$ imply $g_{I}^{\prime}, \ldots, g_{p}^{\prime} \in T$, and, by (5), the submodule generated by $g_{1}^{\prime}, \ldots, g_{r}^{\prime}$ coincides with $T$. So the projective $Z C_{r, n^{-}}$ module $T$ of rank $r$ has $r$ generators. Then $T$ is free. This contradiction shows that $T$ is not free. 


\section{References}

[1] Hyman Bass, Algebraic K-theory (Benjamin, New York, Amsterdam, 1968).

[2] Graham Higman, "The units of group-rings", Proc. London Math. Soc. (2) 46 (1940), 231-248.

[3] David Lissner, "Matrices over polynomial rings", Trans. Amer. Math. Soc. 98 (1961), 285-305.

[4] A.J. Mclsaac, "The freeness of some projective metabelian groups", Bulz. Austral. Math. Soc. (to appear).

[5] Hanna Neumann, Varieties of groups (Ergebnisse der Mathematik und ihrer Grenzgebiete, 37. Springer-Verlag, Berlin, Heidelberg, New York, 1967).

[6] Richard G. Swan, "Periodic resolutions for finite groups", Ann. of Math. (2) 72 (1960), 267-291.

Department of Mechanics and Mathematics,

Moscow State University,

Moscow,

USSR;

Department of Mathematics,

Bedford College,

University of London,

London,

England. 